\title{
Reducing seismic stress on buildings through inertia limiters at floor level
}

\author{
M. C. Porcu \\ Department of Mechanical, Chemical and Materials Engineering, \\ University of Cagliari, Italy
}

\begin{abstract}
A seismic stress control method based on the disconnection of non-structural floor masses during strong earthquakes is investigated in the present paper. Rigid-plastic devices (very stiff in the elastic range, in practice), which may plastically yield when a pre-set level of seismic load is reached, are assumed to connect structural and non-structural floor masses. Such rigid-plastic connectors act as inertia limiters so reducing seismic loads on building floors. In addition, they also act as seismic energy dissipaters due to plastic dissipation which is triggered at the connector level. The method may lead to significant seismic stress reductions. The price to be paid, however, might be large relative displacements of the disconnected mass. By referring to a single-storey frame and to recorded ground motions, a numerical investigation is performed in the present paper showing that the disconnected mass usually experiences rather small relative displacements (generally below $10 \mathrm{~cm}$ ), even when high stress reductions are achieved. For practical purposes, a useful procedure to obtain a quick - though approximate - evaluation of the peak relative displacement of the disconnected mass from the earthquake rigid-plastic pseudo-spectrum is also provided in the paper.
\end{abstract}

Keywords: seismic stress control, rigid-plastic connectors, floor mass disconnection, inertia limiters.

\section{Introduction}

During a ground motion, inertia loads act on building floors. They are proportional to the ground acceleration as well as to the floor mass. In particular, the larger the floor mass is, the higher the inertia loads -and consequently the 
stress on building members- become. Based on disconnecting a portion of active floor mass during strong earthquakes, a method of seismic stress control was proposed in [1] and investigated in [2]. In this method, rigid-plastic devices are interposed between structural and non-structural floor masses. Assumed to be very stiff in the elastic range, such connectors may plastically yield as soon as a pre-set level is reached by the horizontal loads acting on the floor masses. The rigid-plastic connectors, in fact, behave both as inertia limiters since they reduce the active mass and as energy dissipaters since plastic dissipation is involved when they yield. This dual action may reduce - even quite drastically- seismic stress, as also shown in $[1,2]$.

As a quid pro quo, however, high relative displacements of the disconnected mass might be required to achieve substantial reductions in stress, which would be a high price to pay. By referring to a single-story frame and to two strong recorded earthquakes, the present paper investigates this aspect by means of a wide non-linear numerical analysis. It is found that quite low relative displacements are actually experienced by the disconnected mass, even when one half of the floor mass is disconnected and stress reductions of more than $50 \%$ are achieved.

For the purpose of practical application of the method, a simple procedure is provided in the paper which leads to an estimation of the peak relative displacement of the disconnected mass under a given earthquake. This procedure is based on the earthquake rigid-plastic pseudo-spectrum, which is a single curve diagram introduced in [3], providing the peak displacements of rigid-plastic oscillators as a function of the ratio between yield load and mass of the oscillator, cf. [3-7].

It can be noted, finally, that other approaches can be found in the available literature which somehow isolate active mass for the purpose of seismic stress control, cf. e.g. [8-12]. The present method is, however, different from the above-mentioned. Its peculiarity lies in the fact that it reduces the active floor mass as the vibration level reaches certain limits, contemporarily making the disconnected mass work towards the plastic dissipation of seismic energy.

\section{Rigid-plastic disconnection of non-structural floor mass in a single-story frame}

A shear- type single-story frame of mass $M$ and lateral stiffness $k_{l}$ is considered in Figure 1(a) and referred to as Frame A. The maximum stress produced by a given ground motion $\ddot{u}_{g}(t)$ on pillars of this frame can be obtained by applying the following horizontal static load to mass $M$ :

$$
F_{S A}=k_{1} u_{E \max }(T, \xi)
$$

Here $u_{E \max }=u_{E \max }(T, \xi)$ denotes the peak elastic displacement of $M$ under the given earthquake. It is a function both of the natural period $T$ and of the 
damping ratio $\xi$ of the system. The value of $u_{E \max }(T, \xi)$ can be obtained by solving the motion linear equation:

$$
\ddot{u}+\frac{4 \pi \xi}{T} \dot{u}+\frac{4 \pi^{2}}{T^{2}} u=-\ddot{u}_{g}(t) .
$$

In the above equation $u=u(t), \dot{u}=\dot{u}(t)$ and $\ddot{u}=\ddot{u}(t)$ represent displacement, velocity and acceleration of $M$, respectively (all relative to the ground). Of course, when the earthquake response spectrum is available, the value of $u_{E \max }$ can be derived straightforwardly from it.

By taking into account that $T=2 \pi \sqrt{M / k_{1}}$, the seismic load given by equation (1) can also be put in the form:

$$
F_{s A}=\frac{4 \pi^{2}}{T^{2}} M u_{E \max },
$$

which shows the dependence of $F_{S A}$ on the floor mass $M$. In particular, eqn. (3) suggests that a reduction in the floor mass might be beneficial to reduce seismic load and, therefore, seismic stress on the frame. Yet, it should be considered that as $M$ decreases the natural period $T$ of the system decreases too and the value of $u_{E \max }$ might even increase. Therefore, the effectiveness of a reduction in the floor mass for the purpose of stress control needs to be carefully assessed.

To this end, a comparison between the seismic responses of the two frames depicted in Figure 1 may be helpful. The first one, referred to as Frame A, is a linear single-story shear-type frame. Under a given earthquake, its motion is governed by eqn. (2) and the maximum stress on its pillars may be evaluated by applying the static load, given by eqn. (1), to mass $M$.

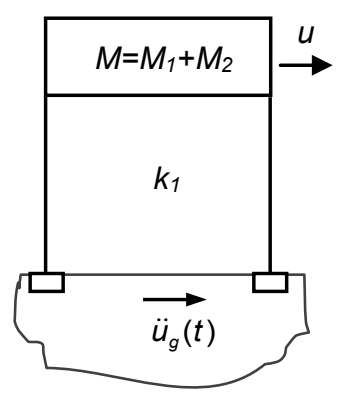

(a) Frame A

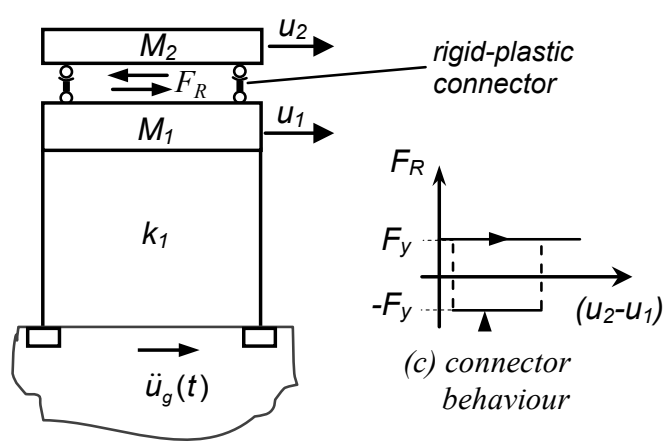

(b) Frame $B$

Figure 1: (a) Shear-type elastic frame; (b) non-linear frame with connectors; (c) rigid-plastic behavior of connectors. 
On the contrary, the second frame in Figure 1, referred to as Frame B, is a non-linear system obtained from Frame $A$ by sharing its total mass $M$ into two portions, say $M_{1}$ and $M_{2}$, and by inserting non-linear connectors between them. The connectors are assumed to be very stiff in the elastic range (practically rigid) and to plastically yield when a pre-set absolute value of force, say $F_{y}$, is reached. The behavior of such connectors is shown in Figure 1c.

Under a given ground acceleration $\ddot{u}_{g}(t)$ the motion equations of Frame $B$ can be written as follows, cf. [2]:

elastic range

$$
\begin{gathered}
\text { if }\left|\ddot{u}_{2}+\ddot{u}_{g}\right|<\frac{F_{y}}{M_{2}} \text { and }\left(\dot{u}_{2}-\dot{u}_{1}\right)=0 \\
\text { or if }\left|\ddot{u}_{2}+\ddot{u}_{g}\right|=\frac{F_{y}}{M_{2}},\left(\dot{u}_{2}-\dot{u}_{1}\right)=0 \text { and } \operatorname{sign}\left(\dot{u}_{2}\right) \neq \operatorname{sign}\left(u_{2}\right) \\
\left\{\begin{array}{l}
\ddot{u}_{1}+\frac{4 \pi \xi}{T} \dot{u}_{1}+\frac{4 \pi^{2}}{T^{2}} u_{1}=-\ddot{u}_{g}(t) \\
u_{2}=u_{1}
\end{array}\right.
\end{gathered}
$$

plastic range:

$$
\begin{aligned}
& \text { if }\left|\ddot{u}_{2}+\ddot{u}_{g}\right|=\frac{F_{y}}{M_{2}} \quad,\left(\dot{u}_{2}-\dot{u}_{1}\right)=0 \quad \text { and } \operatorname{sign}\left(\dot{u}_{2}\right)=\operatorname{sign}\left(u_{2}\right) \text { or if } \\
& \left(\dot{u}_{2}-\dot{u}_{1}\right) \neq 0 \\
& \left\{\begin{array}{l}
\ddot{u}_{1}+\frac{4 \pi \xi_{1}}{T_{1}} \dot{u}_{1}+\frac{4 \pi^{2}}{T_{1}^{2}} u_{1}-\operatorname{sgn}\left(u_{2}\right) \frac{F_{y}}{M_{1}}=-\ddot{u}_{g}(t) \\
\ddot{u}_{2}+\operatorname{sgn}\left(u_{2}\right) \frac{F_{y}}{M_{2}}=-\ddot{u}_{g}(t)
\end{array}\right.
\end{aligned}
$$

In the above equations sign $\cdot \gamma$ denotes the sign of $(\cdot)$, while the meaning of other symbols can be taken from Figure 1(b). Parameters $T_{1}$ and $\xi_{1}$ appearing in the same equations are given by:

$$
\begin{gathered}
T_{1}=2 \pi \sqrt{\frac{M_{1}}{k_{1}}}=T \sqrt{\frac{M-M_{2}}{M}}=T \sqrt{1-\rho_{M}}, \\
\xi_{1}=\frac{\xi}{\sqrt{\frac{M-M_{2}}{M}}}=\frac{\xi}{\sqrt{1-\rho_{M}}},
\end{gathered}
$$


respectively. Here $\rho_{M}$ denotes the ratio between the disconnected mass $M_{2}$ and the total mass $M$. In the following, it will be referred to as the mass ratio. It can be helpful to introduce also the plastic ratio $\rho_{y}$ as defined by, cf. [2]:

$$
\rho_{y}=\frac{F_{y}}{F_{E 2}}=\frac{F_{y}}{M_{2} a_{E \max }(T, \xi)},
$$

where $a_{E \max }=a_{E \max }(T, \xi)$ is the peak elastic acceleration of an oscillator of period $T$ and damping ratio $\xi$ under the given ground motion. The quantity $F_{E 2}=M_{2} a_{E \max }$, in fact, represents the force that the earthquake would exert on mass $M_{2}$ should connection between masses $M_{1}$ and $M_{2}$ be perfectly rigid. Therefore, the plastic ratio $\rho_{y}$ quantifies the plastic involvement of the rigidplastic connectors during the considered earthquake. Since $a_{E \max } \cong \omega^{2} u_{E \max }$, cf. [13], $\omega$ being the natural angular frequency given by $\omega=2 \pi / T$, the plastic ratio may also be expressed as:

$$
\rho_{y}=\frac{F_{y}}{M_{2}} \frac{1}{\omega^{2} u_{E \max }} .
$$

As a function of $\rho_{M}$ and $\rho_{y}$, eqns. (4a)-(4b) may be re-written as:

elastic range

$$
\begin{aligned}
\text { if }\left|\ddot{u}_{2}+\ddot{u}_{g}\right|<\rho_{y} \omega^{2} u_{E \max } \text { or }\left|\ddot{u}_{2}+\ddot{u}_{g}\right|=\rho_{y} \omega^{2} u_{E \max } \text { and } \operatorname{sign}\left(\dot{u}_{2}\right) \neq \operatorname{sign}\left(u_{2}\right) \\
\left\{\begin{array}{l}
\ddot{u}_{1}+\omega \xi \dot{u}_{1}+\omega^{2} u_{1}=-\ddot{u}_{g}(t) \\
u_{2}=u_{1}
\end{array}\right.
\end{aligned}
$$

plastic range:

if $\left|\ddot{u}_{2}+\ddot{u}_{g}\right|=\omega^{2} \rho_{y} u_{E \max },\left(\dot{u}_{2}-\dot{u}_{1}\right)=0$ and $\operatorname{sign}\left(\dot{u}_{2}\right)=\operatorname{sign}\left(u_{2}\right)$ or if $\left(\dot{u}_{2}-\dot{u}_{1}\right) \neq 0$

$$
\left\{\begin{array}{l}
\ddot{u}_{1}+\frac{\omega \xi}{\left(1-\rho_{M}\right)} \dot{u}_{1}+\frac{\omega^{2}}{\left(1-\rho_{M}\right)} u_{1}-\operatorname{sgn}\left(u_{2}-u_{1}\right) \frac{\omega^{2} \rho_{M} \rho_{y}}{\left(1-\rho_{M}\right)} u_{E \max }=-\ddot{u}_{g}(t) \\
\ddot{u}_{2}+\operatorname{sgn}\left(u_{2}-u_{1}\right) \omega^{2} \rho_{y} u_{E \max }=-\ddot{u}_{g}(t)
\end{array}\right.
$$

The above equations show that the response of Frame $B$ to a given earthquake depends on four parameters, namely $\rho_{M}, \rho_{y}, \xi$ and $\omega$ (or $T$ ). It should be considered, in fact, that for a given earthquake, $u_{E \max }(T, \xi)$ is a known quantity which can be derived from the earthquake response spectrum. 
By solving eqns. (8a)-(8b), the maximum displacement $u_{1 \max }$ reached by mass $M_{1}$ in Frame $B$ during the given earthquake can be calculated. As a function of it, the maximum seismic stress on pillars of Frame $B$ can finally be obtained by applying the following horizontal static load to mass $M_{1}$ :

$$
F_{S B}=k_{1} u_{1 \max }\left(T, \xi, \rho_{M}, \rho_{y}\right) .
$$

If $u_{1 \max }$ is lower than $u_{E \max }$, the seismic load $F_{S B}$, as given by eq. (9), will be lower than $F_{S A}$, as obtained from eqn. (1). When this happens the proposed method of plastic disconnection of floor mass becomes effective in reducing seismic stress on frame pillars. By referring to two strong earthquakes and to Frame $A$ and Frame $B$ depicted in Figure 1, a numerical investigation has been performed showing that condition $u_{1 \max }<u_{E \max }$ is generally met. Some results of this investigation are given in Figure 2, where the peak displacements $u_{E \max }$ and $u_{1 \max }$ are plotted together against the natural period $T$ of Frame A. For a more
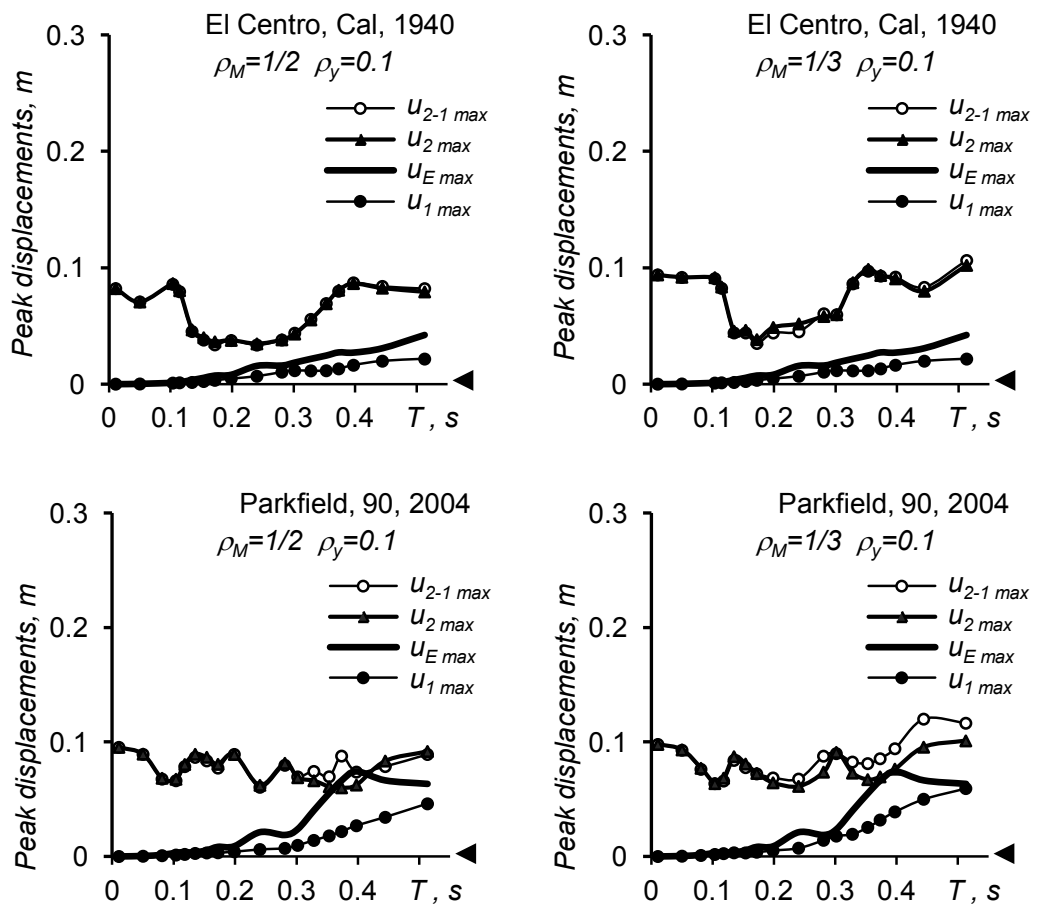

Figure 2: $\quad$ Peak displacements of masses of Frame $A$ and Frame $B$ under two different strong earthquakes, for $\rho_{y}=0.1$ and two values of $\rho_{M}$. 
comprehensive comparison, both the peak displacement of mass $M_{2}$ with respect to the ground, say $u_{2 \max }$, and the peak relative displacement of $M_{2}$ with respect to $M_{1}$, namely $u_{2-1 \max }=\max \left|u_{2}(t)-u_{1}(t)\right|$, are also plotted in Fig. 2 . It can be noted that the values of $u_{2 \max }$ and $u_{2-1 \max }$ are generally very close to each other.

\section{Seismic stress reduction due to plastic mass disconnection}

A comparison between eqn. (1) and eqn. (9) highlights that effective seismic stress control may be achieved as long as the maximum displacement $u_{1 \max }$ of mass $M_{1}$ in Frame $B$ is lower than the maximum displacement $u_{E \max }$ of mass $M$ in Frame A. To assess the effectiveness of the proposed method, therefore, the following parameter can be helpful, cf. [2]:

$$
\Delta_{S}=\frac{F_{S B}-F_{S A}}{F_{S A}}=\frac{u_{1 \max }-u_{E \max }}{u_{E \max }}=\frac{u_{1 \max }}{u_{E \max }}-1 .
$$
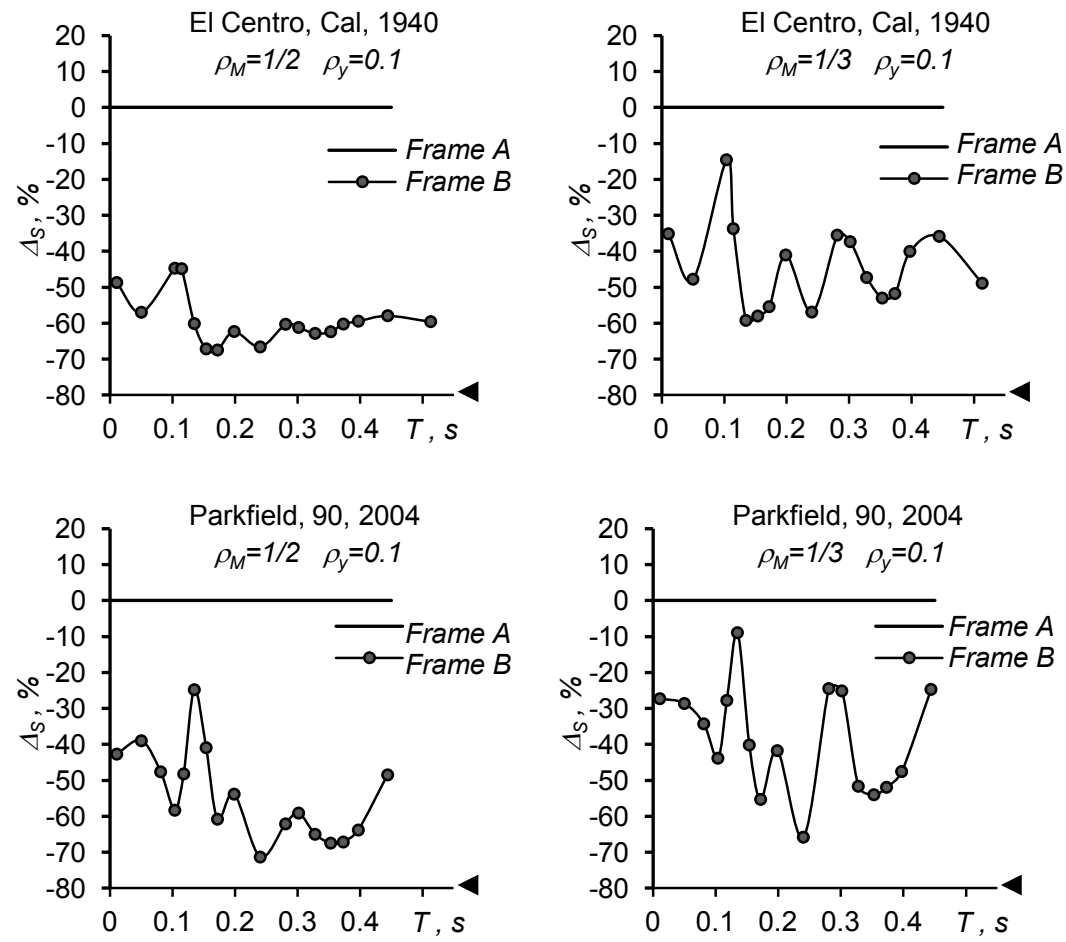

Figure 3: $\quad$ Stress reduction factor versus the natural period $T$ of Frame $A$. The diagrams refer to the same instances considered in Figure 2. 
It gives the reduction in seismic loads. Due to proportionality between loads and stress, it also provides the seismic stress reduction and can be referred to as the stress reduction factor. Should the displacement ratio $u_{1 \max } / u_{E \max }$ be lower than one, the stress factor $\Delta_{F}$ becomes negative, so quantifying, in fact, the stress reduction percentage. Relevant to the same instances presented in Figure 2, the stress reduction factor $\Delta_{S}$ is plotted in Fig. 3 .

Diagrams in Figure 3 show that a stress reduction of up to $55 \%$ may be reached when one third of the floor mass is disconnected $\left(\rho_{M}=1 / 3\right)$ and a low enough plastic limit is set, namely $\rho_{y}=0.1$. Should one half of the mass be disconnected $\left(\rho_{M}=1 / 2\right)$ the seismic stress reduction may even rise to $70 \%$. A more comprehensive collection of results, relevant to other values of $\rho_{y}$ and $\rho_{M}$, may be found in [2].

\section{Peak displacements of the disconnected mass}

Results given in Figure 3 highlight that, generally, the greater the disconnected mass the lower the seismic stress on pillars of Frame $B$ will be. However, as the peak displacement $u_{1 \max }$ of mass $M_{1}$ of Frame $B$ becomes lower with respect to displacement $u_{E \max }$ of mass $M$ in Frame $A$-so reducing seismic stress on pillars- the peak displacement of mass $M_{2}$ is expected, on the contrary, to rise higher and higher. Of course, too high relative displacements of $M_{2}$ would make it inconvenient to apply the present method in practice.

Nonetheless, the results given in Figure 2, together with those given in Figures 5-6, show that the peak relative displacements of mass $M_{2}$ are generally small (lower than 10 centimeters in the considered instances), even when strong earthquakes are considered and high stress reductions are achieved (as can also be inferred by comparing Figure 2 and Figure 3).

\section{Estimating the peak displacements of the disconnected mass from the earthquake rigid-plastic pseudo-spectrum}

To assess in advance whether the present method of rigid-plastic disconnection of floor mass can be advantageously applied in practice, a quick estimate of both the stress reduction which can be achieved and the maximum displacement which will be attained by the disconnected mass $M_{2}$ can be useful. A simple procedure to obtain an estimate of the stress reduction from the earthquake elastic response spectrum is provided in [2]. While, a swift way of estimating the peak relative displacement of mass $M_{2}$ from the rigid-plastic pseudo spectrum of the earthquake will be provided in this section. 
Under a given ground acceleration $\ddot{u}_{g}(t)$, the peak displacement of a rigidperfectly-plastic oscillator of mass $M_{2}$ and yield load $F_{y}$ (absolute value) can be obtained by solving the following motion equations:

$$
\text { if }\left|\ddot{u}_{g}\right|<\frac{F_{y}}{M_{2}} \text { and } \dot{u}_{2}=0 \quad \text { no motion }
$$

if $\quad\left|\ddot{u}_{g}\right| \geq \frac{F_{y}}{M_{2}}$ and $\dot{u}_{2}=0$ or if $\dot{u}_{2} \neq 0 \quad \ddot{u}_{2}-\operatorname{sgn}\left(\ddot{u}_{g}\right) \frac{F_{y}}{M_{2}}=-\ddot{u}_{g}(t)$

Here, $u_{2}=u_{2}(t), \dot{u}_{2}=\dot{u}_{2}(t)$ and $\ddot{u}_{2}=\ddot{u}_{2}(t)$ are relative displacement, velocity and acceleration of mass $M_{2}$. The above equations show that, under a given earthquake, the rigid-plastic motion depends only on the ratio $a_{y}=F_{y} / M_{2}$ which can be referred to as the oscillator yield acceleration, cf. [3-6]. As a function of $a_{y}$, the peak displacements of the rigid-plastic oscillator, say $u_{\max }^{R P}$, may be collected in a diagram called rigid-plastic pseudo-spectrum, cf. [3-7]. The rigidplastic pseudo-spectra of the two earthquakes considered in the present investigation are given in Fig. 4 (where $g$ denotes the gravity acceleration).
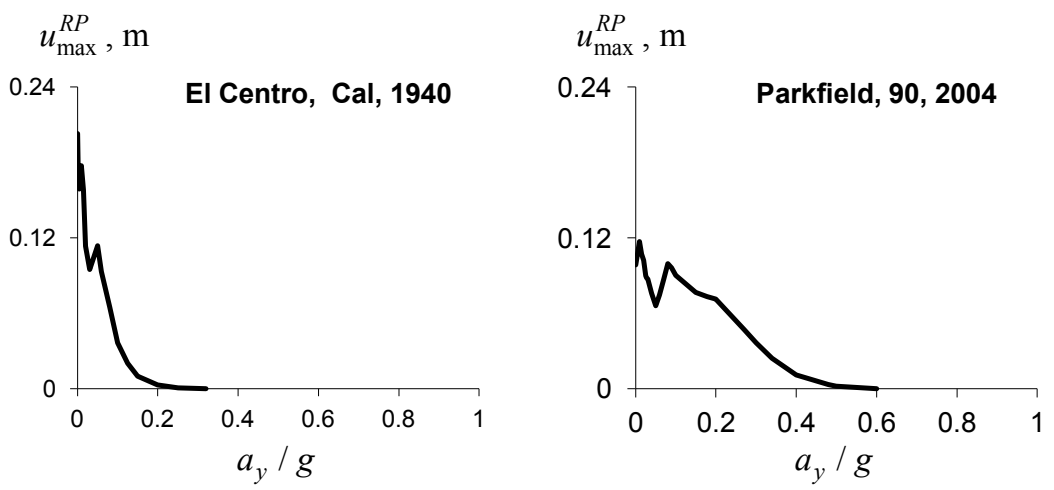

Figure 4: Rigid-plastic pseudo-spectra of the considered earthquakes.

The peak displacement $u_{\max }^{R P}$ as taken from the rigid-plastic pseudo-spectrum for a given $a_{y}$, can be exploited to estimate the peak relative displacement $u_{2-1 \max }=u_{2-1 \max }\left(\rho_{y}, \rho_{M}, T, \xi\right)$ of mass $M_{2}$ of Frame $B$. In view of eq. (7b), in fact, $a_{y}$ can also be put as a function of $\rho_{y}, T$ and $u_{E \max }(T, \xi)$ :

$$
a_{y}=\rho_{y} \frac{4 \pi^{2}}{T^{2}} u_{E \max }(T, \xi) .
$$


For each given value of $a_{y}$, the value of $u_{2-1 \max }$ may be estimated as follows:

$$
\begin{array}{cc}
\text { for } T<0.3 s & u_{2-1 \max }=u_{M A X}^{R P}\left(a_{y}\right) \\
\text { for } T \geq 0.3 s & u_{2-1 \max }=u_{M A X}^{R P}\left(a_{y} / 2\right)
\end{array}
$$
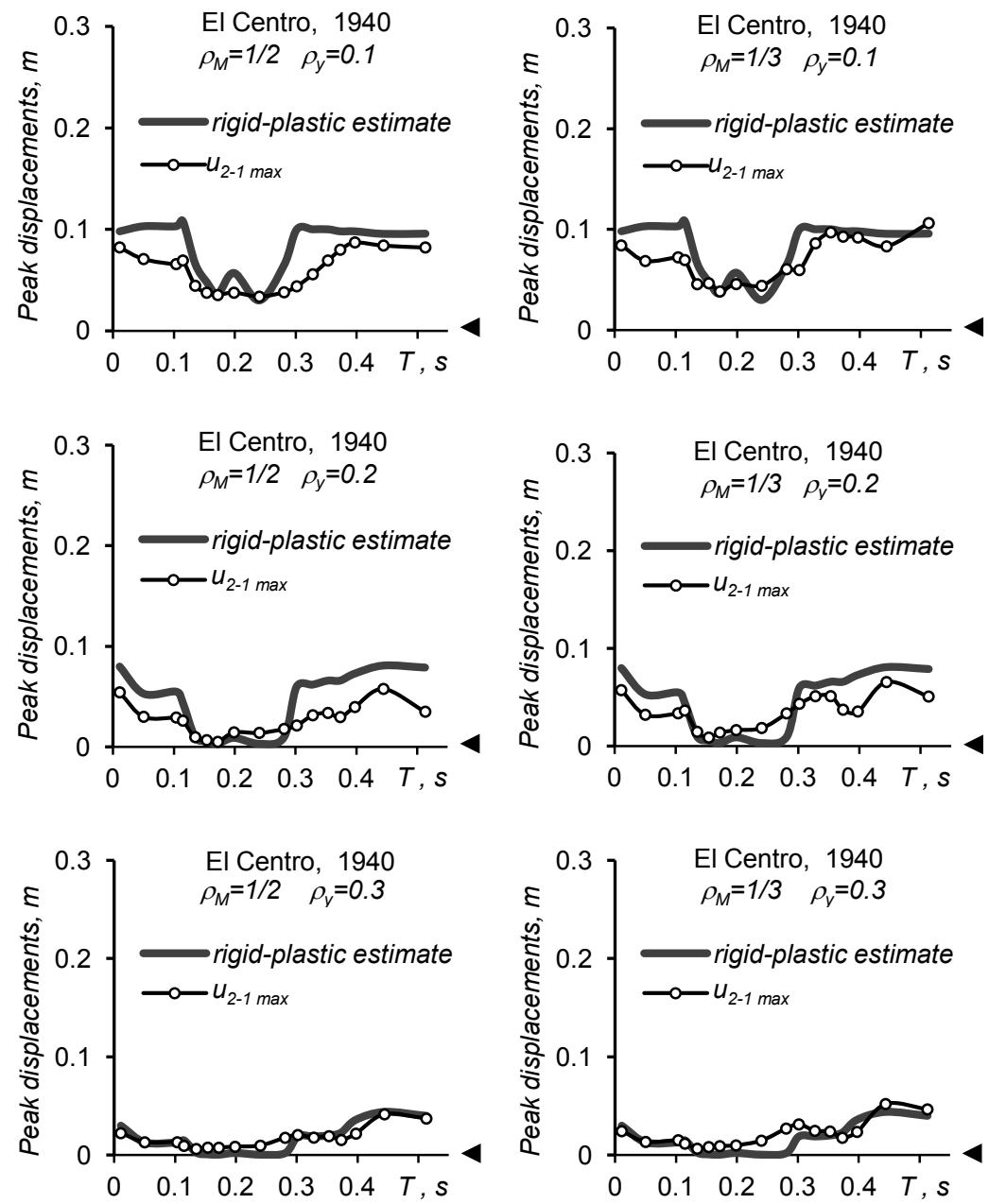

Figure 5: Calculated and estimated peak relative displacements of mass $M_{2}$ under the $1940 \mathrm{El}$ Centro earthquake. The estimated values are obtained from the rigid-plastic spectrum, by applying eqns (14a)(14b). 
Figures 5-6 show that the rigid-plastic estimate, as obtained from the earthquake rigid-plastic pseudo-spectrum by means of relations (14a)-(14b), is rather good, whatever the earthquake, the value of $\rho_{y}, \rho_{M}$ or $T$.
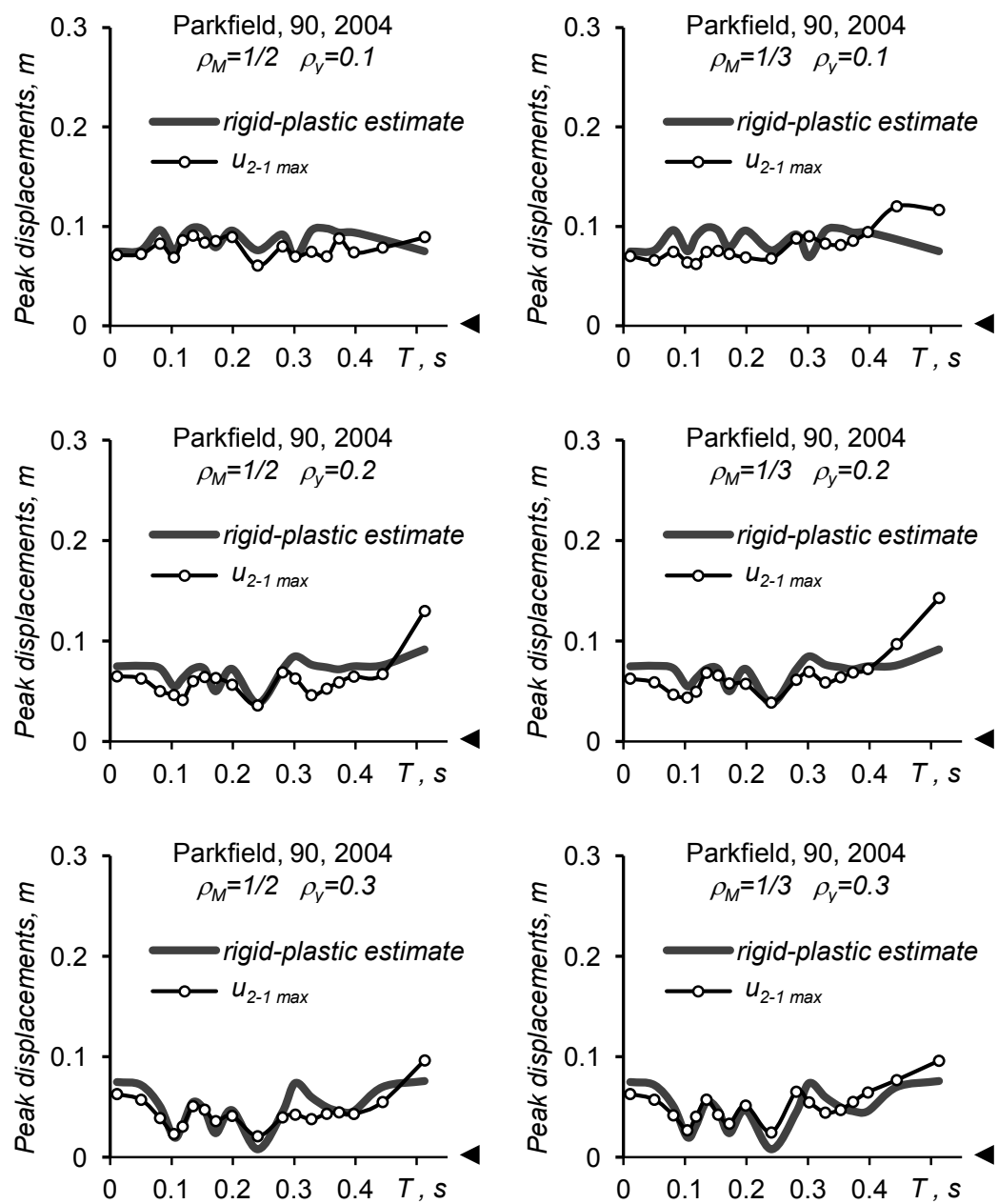

Figure 6: Diagrams analogous to those in Figure 4, but relevant to the Parkfield 2004 earthquake.

\section{Conclusions}

A seismic stress control method based on the rigid-plastic disconnection of building non-structural floor masses is assessed in the paper. By means of a nonlinear numerical analysis, the displacement demand on the disconnected mass of a single-story frame is assessed for different earthquakes. The results show that, 
despite the drastic stress reduction which may be achieved, rather small relative displacements are experienced by the disconnected mass (generally lower than $10 \mathrm{~cm}$ in the considered instances), whatever the period of vibration of the frame, the plastic limit of the rigid-plastic connectors, the percent of mass disconnected or the violence of the earthquake. For practical purposes, a simple way of obtaining a good prediction of the peak displacement of the disconnected mass from the earthquake rigid-plastic pseudo-spectrum is provided in the paper.

\section{References}

[1] Paglietti A. and Porcu M.C., Controlling Dynamic Stress through Plastic Limiters, Proceedings of the 1st European Conference on Structural Control, A. Baratta and J. Rodelar (eds), Barcellona, Spagna, World Scientific Publishing, Singapore, pp. 483-490, 1996.

[2] Porcu M.C., Plastic Disconnection of Non-Structural Floor Masses to Reduce the Dynamic Response of Buildings. To appear.

[3] Paglietti A. and Porcu M. C., Rigid-plastic approximation to predict motion under strong earthquakes, Earthquake Engineering and Structural Dynamics, 30, pp. 115-126, 2001.

[4] Porcu M.C. and Mascia M., Rigid-Plastic Pseudo-Spectra: Peak Response Charts for Seismic Design, European Earthquake Engineering, 3, pp. 3747, 2006.

[5] Porcu M.C. and Carta G., A better rigid-plastic estimate for earthquakeinduced plastic displacements, Int. J. of Safety and Security Engineering, 2(2), pp. 184-196, 2012.

[6] Porcu M.C. and Carta G., Rigid-Plastic Seismic Analysis to predict the structural ductility demand, International Journal of Applied Engineering Research, 4(3), pp. 309-325, 2009.

[7] Domingues Costa JL, Bento R, Levtchitch V, Nielsen MP (2007), Rigidplastic seismic design of reinforced concrete structures, Earthquake Engineering and Structural Dynamics, 36, pp. 55-76.

[8] Kondo H., Floor system for seismic isolation, Patent number 4,662,133, United States, 1987.

[9] Su L., Ahmadi G. and Tadjbakhsh I.G., Comparative Study of Base Isolation Systems, Journal Engineering Mechanics, 115(9), pp.1976-1992, 1989.

[10] Villaverde R., Roof Isolation System to Reduce the Seismic Response of Buildings: A Preliminary Assessment, Earthquake Spectra, 14(3), pp.521532, 1998.

[11] Ziyaeifar M., Method of Mass Isolation in Seismic Design of Structures, Proc. of the 12th World Conference on Earthquake Engineering, 2000.

[12] Ziyaeifar M., Noguchi H., Partial Mass Isolation in Tall Buildings, Earthquake Engineering \& Structural Dynamics, 27, pp.49-65, 1998.

[13] Chopra A.K., Dynamics of Structures, Prentice-Hall, New Jersey, 2001. 